\title{
2 La lingüística cognitiva: un nuevo paradigma
}

La lingüística cognitiva (LC) no es una teoría lingüística claramente determinada sino una estrategia de estudio del lenguaje (Croft \& Cruse, 2004) en la que convergen un conjunto heterogéneo e interdisciplinar de estudios que tienen como punto de contacto una perspectiva sobre el lenguaje y la cognición.

Los inicios de la LC se pueden ubicar grosso modo alrededor de 1975, cuando George Lakoff usa el término por primera vez. Además, Charles Fillmore por esos años comienza a trabajar en la semántica de marcos (Frame semantics), Ronald Langacker en la Space Grammar que más tarde adquirirá el nombre de Cognitive Grammatics y Leonard Talmy aplica conceptos de la psicología de la Gestalt, tales como figura/fondo, al análisis lingüístico.

Este amplio conjunto de investigaciones que se desarrollaron a finales de los años 70 y durante los 80 consideran el lenguaje como una de las manifestaciones de una capacidad cognitiva más general que permite conectar las diferentes informaciones recibidas y almacenadas en la memoria. Uno de los principios de los que parten estas investigaciones es la idea de que el lenguaje es un sistema simbólico que refleja la conceptualización de la experiencia tanto lingüística como no lingüística y que permite almacenarla.

Para esta perspectiva todos los sistemas cognitivos, incluida la lengua, existen y funcionan debido a que su propia organización permite la interacción entre ellos. El lenguaje no es una facultad autónoma sino una capacidad cognitiva que depende de otros procesos mentales y, por tanto, está intrínsecamente ligado a procesos cognitivos básicos como la percepción, la atención y la categorización.

El segundo principio compartido por los estudios que conforman la LC es la idea de que la naturaleza conceptual del significado no puede reducirse a una simple relación de carácter veritativo-condicional con el mundo real, sino que es el resultado de un proceso cognitivo. Cualquier experiencia que se quiere comunicar proviene tanto de una organización conceptual, o sea, de sistemas de conocimiento almacenado (que operan de manera offline), así como de una conceptualización, es decir, de procesos cognitivos de construcción de significado que se llevan a cabo sobre la marcha del discurso (online) (Hart 2010, pp. 24-25).

La LC representó una revolución en el ámbito de las ciencias cognitivas. Sostuvo un punto de vista experiencial que se contraponía a la idea objetivista de la mente separada del cuerpo. Este es el tercer punto en el que convergen las contribuciones en LC, esto es, según este enfoque, la mente interactúa con el ambiente y con el cuerpo, y tal interacción estructura nuestras operaciones mentales. El pensamiento ya no es visto bajo la óptica objetivista como un procesamiento de símbolos abstractos que obtienen significado en corresponden- 
cia con el mundo exterior, sino que surge de la experiencia corpórea y adquiere sentido a través de esta (en inglés embodiment, que se refiere al carácter corpóreo del lenguaje). En suma, para la LC nuestros sistemas conceptuales están basados en la percepción, el movimiento corporal y en la experiencia física y social.

Finalmente, otra de las ideas principales propias de la LC es que el conocimiento del lenguaje está ligado al uso, en el sentido de que la adquisición de todas las estructuras lingüísticas proviene de la experiencia. En este sentido, Tomasello (2005, p. 175) afirma que la adquisición de una lengua depende de la memorización de pequeñas instancias y se desarrolla a través de la experiencia lingüística de cada hablante. En efecto, este enfoque estudia la lengua partiendo de la parole, o sea, del uso que el hablante hace de la lengua, y ya no de la langue, la lengua vista como sistema abstracto, como lo hacen las lingüísticas estructuralista y generativa.

\subsection{La organización conceptual: marco, dominio y modelo cognitivo idealizado}

La LC surge como reacción a los enfoques formalistas de la gramática estructuralista y generativa. Mientras que esta última considera la lengua como un dispositivo autónomo que produce frases bien formadas, la LC, en contraste, parte de la idea de que el lenguaje es un sistema simbólico no autónomo que refleja la conceptualización de la experiencia lingüística y no lingüística. Por esta razón, la atención de los lingüistas cambia hacia la semántica: ya no será la sintaxis el núcleo generador de la gramática, sino la semántica, ya que, de acuerdo con este enfoque, tanto la morfología como la sintaxis están motivadas por esta.

Por otro lado, la semántica estructuralista sostiene que las palabras denotan conceptos - considerados como unidades de significado-, analiza las relaciones semánticas entre estos (hiponimia, hiperonimia, antonimia, sinonimia, etc.), sostiene que los conceptos pueden descomponerse en rasgos semánticos y formar parte de una categoría si cumple con las propiedades necesarias y suficientes. Sin embargo, la teoría de los rasgos semánticos resulta insuficiente para explicar cómo se relacionan los conceptos. Para la LC la categorización se produce de manera diferente y no existen categorías cerradas ni límites precisos entre ellas. Además, las palabras no están dispersas al azar en la mente. Las palabras están relacionadas unas con otras -como sostiene la semántica estructural-, pero estas relaciones no son solo de tipo semántico, sino que hay conceptos que están ligados a otros debido a que están asociados a la experiencia humana cotidiana. Apoyándonos en el ejemplo clásico de Schank y Abelson 
(1977; 1987), el concepto de [RESTAURANTE] está asociado con muchos otros como [COMENSAL], [CAMARERO], [ORDENAR], [COMER], [CUENTA], [PAGAR], [PROPINA], etc. Dichos conceptos no están asociados a [RESTAURANTE] por relaciones semánticas de tipo estructural, sino por la experiencia humana común.

\section{A. La organización conceptual a través de marcos}

Se han formulado distintas propuestas sobre la manera en que se relacionan los conceptos y se han ofrecido diversas denominaciones parecidas sustancialmente. Una de las propuestas más relevantes de la LC ha sido la semántica de marcos de Charles Fillmore (1985). Esta sostiene que tras la pronunciación de un enunciado el oyente evoca un determinado marco para poder comprender el input lingüístico. Fillmore (2006, p. 374) define marco de la siguiente manera:

\footnotetext{
By the term 'frame' I have in mind any system of concepts related in such a way that to understand any one of them you have to understand the whole structure in which it fits; when one of the things in such a structure is introduced into a text, or into a conversation, all of the others are automatically made available.
}

Un marco o frame es una esquematización de la experiencia que se almacena en la memoria y que une, como en una red, diferentes conceptos relacionados. Una palabra sirve para abrir el marco que está asociado a ella, pero solo permite focalizar la atención del hablante y del oyente en una parte del frame (Taylor 1999, p. 189); es decir, ninguna palabra puede reflejar la estructura completa de este. Otro aspecto importante de los marcos es que muchos conceptos no pueden ser entendidos si no se conocen entidades externas como la intención del hablante o las convenciones y comportamientos sociales/culturales en el que se sitúa la palabra o que circundan el acto comunicativo. Por ejemplo, el concepto [VEGETARIANO] tiene sentido solo si se tiene internalizado el frame de una cultura en la cual es común el consumo de carne (Croft \& Cruse 2004, p. 11).

La semántica de marcos resulta eficaz para dar explicación a la comprensión del texto, ya que, por ejemplo, es útil para el análisis de la coherencia. Así, citando un ejemplo de Fillmore (1977, p. 75), de los enunciados Ayer tuve problemas con el coche. El cenicero estaba sucio, el segundo es incoherente con relación al primero, ya que el cenicero (aun cuando se le pueda relacionar con COCHE), no se encuentra dentro del marco tener problemas con el coche. Además, la teoría de los marcos permite enfrentar muchas cuestiones del significado que serían problemáticas si se quisieran explicar mediante la semántica de tipo formal. Por ejemplo, algunos conceptos parecerían referir a una misma entidad y son clasificados como equivalentes en términos veritativo-funcionales, sin embargo, 
hay diferencias de significado que se deben a diferencias de marco. Fillmore (2006, p. 382) cita el ejemplo de [LAND] (tierra) y [GROUND] (suelo) que en inglés perfilan algo que parece ser lo mismo, pero en marcos diferentes: [LAND] describe la superficie seca de la tierra en contraste con [SEA] (mar), mientras que [GROUND] describe la superficie seca de la tierra en contraste con [AIR] (aire). El marco elegido por una palabra o por la otra permite hacer inferencias distintas, por lo que un ave que spends its life on land es un ave que no se mete al agua, mientras que un ave que spends its life on the ground es un ave que no vuela. Estos ejemplos ilustran que los términos se pueden perfilar en marcos diferentes y, como hemos mencionado arriba, de acuerdo al marco seleccionado se evocan diferentes inferencias.

\section{B. La relación perfil-base/dominio}

Como se mencionó anteriormente, se han propuesto distintos términos para referirse al sistema conceptual en el que se organizan y relacionan los significados. Varios de estos términos como el de marco de Fillmore (1977, 2006 [1982]), el de base o dominio propuesto por Langacker (1987) o el de modelo cognitivo dealizado de Lakoff (1987) han sido utilizados de manera intercambiable. Sin embargo, no representan exactamente lo mismo (Langacker 2008, p. 46), tal y como ilustraremos a continuación.

De acuerdo con Langacker $(1987, \S 5.1)$ una expresión adquiere su significado imponiendo un perfil a una base. El autor usa el ejemplo de los conceptos RADIO y CíRCULO para demostrar que solo resulta posible entender el significado de RADIO (perfil) a partir de una comprensión previa del concepto de CíRCULO (base o dominio). En este sentido los dos conceptos están íntimamente asociados y su relación se representa en una estructura conceptual. El perfil hace referencia al concepto simbolizado por la palabra. La base a aquel conocimiento o estructura conceptual que se presupone a partir del perfil y se evoca con el fin de comprender una expresión determinada. Además, una base generalmente incluye una gran variedad de perfiles conceptuales, tomando el primer ejemplo, la base CírCULO engloba no solo RADIO, sino también CIRCUNFERENCIA, DIÁMETRO, CENTRO, etc. Es importante señalar que en la gramática cognitiva se emplea el verbo perfilar (profiling) para referirse al proceso de selección de un determinado aspecto (perfil) dentro de una base (dentro de la estructura conceptual que lo contiene). Así, por ejemplo, RoDILLA perfila una subestructura de la estructura de la base PIERNA. 
Langacker habla de dominio en términos muy semejantes al de base: ${ }^{1}$

A context for the caracterization of a semantic unit is referred to as a domain. Domains are necessary cognitive entities: mental experiences, representational spaces, concepts, or conceptual complexes (Langacker 1987, p. 147).

What is a domain, exactly? To serve its purpose, the term is broadly interpreted as indicating any kind of conception or realm of experience (Langacker 2008, p. 44).

Como se puede observar, las explicaciones de lo que son los dominios son muy similares a la definición de marco de Fillmore. Sin embargo, la teoría de los dominios de Langacker es más articulada y de acuerdo con Evans y Green (2006, pp. 230-231), complementa en varios aspectos a la de la semántica de marcos. Por ejemplo, la teoría de Langacker estipula que una expresión puede presuponer simultáneamente una serie de dominios cognitivos que sirven de base de fondo para construir su significado. A este conjunto de dominios lo llama dominios matriz (1987, p. 147, 2008, pp. 44, 47). Así, por ejemplo, Langacker (2008, p. 47) utiliza la expresión vaso (glass) para explicar esta noción: según nuestra experiencia, los dominios que esta expresión evoca son los de espacio, forma (típicamente cilíndrica), orientación espacial (vertical), función (contenedor de líquidos), función en el proceso de beber (tomar con las manos, ingestión, etc.), material (típicamente vidrio), tamaño (fácilmente sostenible con la mano). Además, según Langacker, algunos dominios son más centrales que otros:

Certain domains are so central that we can hardly use the expression without evoking them, some are activated less consistently, and others are so peripheral that we invoke them only in special circumstances, when they happen to be relevant (2008, p. 48).

En segundo lugar, la teoría de Langacker (1987, pp. 148-150) alude a dos niveles de organización conceptual a través de la distinción entre dominios básicos (basic domains) y dominios abstractos (abstract domains). Los primeros dominios son aquellos que derivan de la experiencia corpórea humana (embodiment) y que no presuponen otros dominios, por ejemplo, el ESPACIO, la MATERIA, el TIEMPO, la FUERZA y las sensaciones perceptivas y corporales como el COLOR, la DUREZA, la INTENSIDAD SONORA, el HAMBRE, el DOLOR, etc. Los dominios abstractos son aquellos dominios que no son básicos, es decir, son conceptos o estructuras conceptuales más complejas que funcionan de base para la definición de otros conceptos (Langacker 1987, p. 150). Además, en la teoría de Langacker (2008, p. 64) los dominios se organizan de manera jerárquica. Esto significa que un

1 Langacker (2008, p. 66) se refiere a base en estos términos: "As the basis for its meaning, an expression selects a certain body of conceptual content. Let us call this its conceptual base”. 
dominio más bajo en la jerarquía es presupuesto por el/los dominio/s de mayor jerarquía, por ejemplo, el dominio FALANGES es perfilado con respecto al dominio MANO que a su vez presupone el dominio BRAZO contenido en el dominio CUERPO, que es entendido en el dominio ESPACIO (el cual es un dominio básico).

\section{El modelo cognitivo idealizado}

Otro constructo conceptual que suele ser intercambiable con dominio o marco y que nos será útil para el desarrollo de este trabajo es el de modelo cognitivo idealizado (a partir de aquí MCI) noción, introducida dentro de la lingüística cognitiva por Lakoff (1987, pp. 68-71). Los MCI son estructuras mentales de entidades y conceptos. Son construidas a partir de la experiencia (la cual incluye también el discurso), se almacenan en la memoria semántica (van Dijk 2003, p. 21) y son utilizadas, a su vez, en la conceptualización de más experiencia (Hart 2011, p. 179). Se trata de conocimientos interrelacionados a partir de los cuales entendemos y formulamos conceptos, pero son idealizados en tanto que son abstracciones que no hacen referencia a entidades concretas y, por lo tanto, no necesariamente se ajustan a la realidad:

[. . .] we organize our knowledge by means of structures called idealized cognitive models, or ICMs, [. . . ] category structures and prototype effects are by-products of that organization (Lakoff 1987, p. 68). ${ }^{2}$

An idealized cognitive model may fit one's understanding of the world either perfectly, very well, pretty well, somewhat well, pretty badly, badly, or not at all (Lakoff 1987, p. 70).

Una forma léxica evoca un conjunto de asociaciones y conocimientos que surgen de los contextos prototípicos en que dicha forma léxica se emplea. Lo anterior permite que para una forma haya más de una representación mental con distintos niveles de complejidad. De ahí que existan cluster models (Lakoff 1987, p. 74), conceptos complejos formados por la aglutinación de varios MCI. Este complejo es psicológicamente más básico que los modelos que lo conforman (1987, p. 74). Lakoff ejemplifica el aglutinamiento de modelos con la palabra madre y afirma que

2 De acuerdo con Rosch (1978) los efectos de prototipicidad son asimetrías entre los miembros de una categoría considerados como mejores o peores representantes de esta. Es decir, un miembro de la categoría estará más al centro de esta cuanto más se parezca al prototipo, por el contrario, mientras más difiera, será un miembro más periférico. En este sentido, la pertenencia a una categoría es una cuestión de grado (ver infra). 
para una comprensión adecuada del término es necesario considerar al menos cinco dominios o modelos cognitivos (además de aquellos de [SER HUMANO] y [MUJER] (1987, pp. 74-6):

- El modelo del NACIMIENTO: una madre es una mujer que da a luz un niño.

- El modelo GENÉTICO: una madre es una mujer que aporta el material genético a un niño.

- El modelo de la CRIANZA: una madre es una mujer adulta que cría y educa al niño.

- El modelo conyUgaL: la madre es la mujer del padre.

- El modelo GENEALÓGico: una madre es el antepasado femenino más próximo.

Los cinco modelos o dominios contribuyen a crear lo que entendemos como el concepto prototípico de madre, que sería la mujer que tiene todos estos elementos. Sin embargo, solo algunas partes del modelo para MADRE pueden ser aplicables a individuos reales, es decir que dentro de la categoría MADRE se puede colocar a otro tipo de mujeres que, aunque no cumplen con todos estos modelos, siguen formando parte en esta categoría. Por ejemplo, una madre adoptiva no cumple el modelo NATAL, una madre soltera no cumple el modelo MARITAL, o una madre por subrogación cumple el modelo NATAL y solo en algunos casos el GENÉTICO, pero, aún así, siguen estando incluidas en esta categoría, aunque con distintos grados de prototipicidad. De ahí que el MCI marque las pautas para determinar al prototipo.

Como hemos tratado de dilucidar, los términos dominio, marco y $\mathrm{MCI}$ se refieren a complejos de conocimientos que son implicados por un concepto. Aunque parecen muy similares y se han utilizado de manera indistinta, no son exactamente equivalentes. En palabras de Langacker (2008, pp. 46-47):

Domain has the greatest generality, since neither frame nor ICM applies very well to basic domains (e.g. time or color space). A frame may be roughly comparable to a nonbasic domain.

En suma, para los fines que nos hemos fijado, proponemos entender marco como una red de conocimientos que son evocados por los conceptos y que permiten su comprensión. Esta red comprende informaciones en gran medida compartidas, pero también incluye experiencias, emociones y conocimientos personales. Dominio refiere a una estructura más pequeña, esto es, la porción mínima de conocimiento necesaria para entender un concepto. Los MCI, por su parte, son redes de distintos modelos cognitivos que en conjunto definen al prototipo. 


\subsection{Operaciones de conceptualización}

Como apunta el segundo principio de la LC que anotamos más arriba, todos los elementos del discurso, incluidos los elementos gramaticales, implican un proceso de conceptualización. De acuerdo con Langacker (1991, p. 294, 2008, p. 4) hay una infinidad de maneras en las que se puede representar un evento y la lengua cuenta con diversos medios para representar una misma situación. Esta capacidad de concebir la misma escena de diferentes maneras es a lo que Langacker llama en inglés imagery (1987, p. 110) o construal (2008, p. 43). En LC, a esta diversidad de medios se les conoce como operaciones de conceptualización u operaciones de estructuración conceptual (construal operations).

Son numerosas las operaciones de conceptualización identificadas por los lingüistas cognitivos y se han propuesto también diferentes modos de clasificarlas. Entre las clasificaciones más conocidas se encuentra la de Talmy (1977, 1978, 1988) y la de los ajustes focales (focal adjustments) de Langacker (1987). Sin embargo, estas clasificaciones no comprenden algunos procesos cognitivos que pueden ser considerados como operaciones de estructuración conceptual. Por ejemplo, ni el enmarcado de Fillmore ni la teoría de la metáfora de Lakoff y Johnson aparecen en las clasificaciones de Talmy ni en la de Langacker.

Croft y Cruse (2004) proponen una clasificación más extensa de las operaciones de conceptualización, cuya novedad radica en que muestran la relación que existe entre las operaciones de conceptualización propuestas por los lingüistas y los procesos mentales distinguidos por los psicólogos cognitivos. En este sentido, los autores (2004, p. 45) afirman que "If linguistic construal operations are truly cognitive, then they should be related to, or identical with, general cognitive processes that are postulated by psychologists".

Según el análisis de Croft y Cruse (2004), las distintas operaciones de conceptualización son manifestaciones de las cuatro capacidades cognitivas básicas mediante las que procesamos la experiencia y que nos permiten hacer una elección entre los distintos modos de representar una escena: la atención/prominencia, la comparación/juicio, la perspectiva/situacionalidad y la constitución/Gestalt (forma).

Para el análisis que ofrecemos en este trabajo nos serviremos solo de algunas de las operaciones de conceptualización clasificadas por Croft y Cruse (2004, p. 46), las cuales serán descritas a continuación. 


\subsubsection{La atención/selección y sus operaciones: la metonimia y el ajuste escalar}

Como explican Croft y Cruse (2004, p. 47), existen ciertas propiedades de los fenómenos que atraen más nuestra atención que otros, es decir, que tienen mayor prominencia (salience). Tal capacidad se encuentra en todos los dominios del pensamiento, pero Croft y Cruse (2004, p. 47) la explican paragonándola con la capacidad visual de los seres humanos. En efecto, cuando vemos una escena seleccionamos ciertos objetos sobre los cuales fijamos la atención; podemos concentrarnos en un punto sin olvidar del todo el ámbito que lo rodea; vemos la escena con mayor o menor detalle; fijamos la mirada o recorremos toda la escena.

La operación conceptual de selección es una de las manifestaciones de la habilidad cognitiva de atención. La selección es el ajuste focal (Langacker 1987) que permite elegir las partes de la experiencia que son relevantes para alcanzar nuestro objetivo e ignorar los aspectos irrelevantes. Ejemplos de selección son la elección de un marco o dominio semántico en el cual se perfila un concepto, o de los elementos/facetas relevantes del marco que permitirán entender tal concepto.

\section{A. La metonimia}

La metonimia es otro ejemplo de selección. Para la LC, la metonimia es la habilidad de seleccionar, dentro de un mismo dominio, otro perfil diferente en términos contextuales al que habitualmente simboliza la palabra (Langacker 2008, p. 69).

Algunos lingüistas cognitivos consideran que toda metonimia, por definición, tiene una función referencial (cfr. Lakoff \& Johnson [1980] 1986, p. 74; Croft 2002), según la cual una entidad está por otra (A por B) dentro del mismo dominio cognitivo y es un punto de referencia que activa o da acceso a otra entidad (cfr. Langacker 2008, p. 504; Kövecses 2002, p. 45). Sin embargo, también se ha considerado que, aunque la metonimia suele ser referencial, existen casos en los que no lo es ${ }^{3}$ (Taylor 1999 [1995]; Ruiz de Mendoza \& Otal Campo 2002; Barcelona 2005). Además, se ha propuesto que, al igual que en la metáfora (ver infra), la metonimia comprende la correspondencia o proyección (mapping, $c f r$.

\footnotetext{
3 Una metonimia es referencial cuando se usa un sintagma nominal (SN) para señalar o referirse a una entidad externa (Barcelona 2016). Por ejemplo, la metonimia iEse Mercedes es un idiota! Debería ser detenido por exceso de velocidad es una metonimia referencial porque Ese Mercedes se refiere a una entidad individual, es decir, al conductor del coche (Barcelona 2005, p. 111). En cambio, la metonimia Pedro es un cerebro no es referencial porque el SN un cerebro no refiere a una entidad, sino que expresa una cualidad atribuida al referente Pedro (Barcelona 2016, p. 124).
} 
Lakoff \& Turner 1989) entre un dominio meta (target domain) y un dominio fuente (source domain) pertenecientes ambos a un mismo dominio matriz:

Metonymy is an asymetrical mapping of a conceptual domain, the source, onto another domain, the target. Source and target are in the same functional domain and are linked by a pragmatic function, so that the target is mentally activated (Barcelona 2005, p. 110).

Barcelona (2005, p. 110; 2016, p. 127) explica que la metonimia es una proyección porque la fuente es un punto de referencia conceptual (cfr. Langacker 1987) que causa la activación mental de la meta. Si bien hay una correspondencia entre dominios, este proceso no es simétrico como en la metáfora, ya que a cada elemento del dominio fuente no corresponde un elemento análogo en el dominio conceptual meta. En la metonimia, las características prominentes de un dominio entero pueden ser proyectadas a un subdominio o al revés, las características prominentes de un subdominio pueden ser proyectadas al dominio entero. Para explicar esto, Dirven (2005, p. 23) pone el ejemplo de la metonimia no referencial María es una cara bonita, donde el subdominio fuente cara bonita es proyectado en el dominio meta María. Es decir, el dominio fuente está encapsulado en el dominio meta. A este tipo de metonimia se le llama source-in-target. El caso contrario lo ejemplifica con la metonimia Bush atacó Irak, donde el dominio fuente Bush está por el subdominio meta el ejército norteamericano. Este tipo de metonimia se denomina target-in-source metonymy porque el dominio meta está comprendido dentro del dominio fuente.

Otro aspecto de la definición de metonimia de Barcelona (2005) es que este proceso se lleva a cabo dentro de un mismo dominio cognitivo funcional y no taxonómico. Para el lingüista, un dominio funcional es equivalente al término de frame de Fillmore (1985) o al de modelo cognitivo idealizado de Lakoff (1987). En este sentido, explica que La Casa Blanca está lista para atacar es una metonimia, ya que el edificio presidencial y los funcionarios que allí trabajan pertenecen a diferentes dominios taxonómicos, pero comparten el mismo dominio funcional o marco (el dominio LOCATIVO) (Barcelona 2005, p. 110). Con respecto a la función pragmática mencionada en su definición, esta tiene que ver con la concepción de la metonimia de Lakoff y Johnson (1980, p. 77), quienes dicen que "[. . .] como los conceptos metafóricos, los metonímicos se fundan en nuestra experiencia”. Es decir, Barcelona explica que una propiedad fundamental de este proceso conceptual es el hecho de que el dominio fuente se proyecta en el dominio meta y lo activa en virtud de un vínculo experiencial (por lo tanto, pragmático) que une ambos dominios dentro del mismo dominio funcional. Además, explica el autor (2016, pp. 129-130) que la función pragmática que conecta los dominios fuente y meta puede ser más o menos fuerte, esto es, su fuerza depende de la cercanía conceptual entre estos dos dominios: si fuente y meta están conceptualmente ale- 
jados, el enlace será débil y viceversa. El grado de fuerza del enlace pragmático, pues, favorecerá la comprensión y convencionalización de las expresiones metonímicas.

\section{B. El ajuste escalar}

Como habíamos dicho, existen diferentes maneras de conceptualizar una escena. Al enfocarse mayor o menormente en ciertos aspectos y, por lo tanto, al organizar la escena lingüísticamente de una manera específica, el hablante impone una interpretación única. En este sentido, un tercer aspecto de la atención es el ajuste de la escala de atención (scalar adjustement, Talmy 1983, p. 238). Existen dos tipos de ajustes focales (Croft y Cruse 2004, pp. 52-53), uno cuantitativo y uno cualitativo. El primero coincide con el ajuste focal que Langacker nomina abstracción (1987) y tiene que ver con el grado de especificidad con el que se construye una escena. Basándonos en los ejemplos de Talmy (1983, p. 238): (a) Ella cruzó corriendo por el campo y (b) Ella corrió a través del campo, podríamos decir que describen la misma escena; sin embargo, el ejemplo (b), mediante la preposición a través se resalta la espesura de la vegetación, ya que esta preposición exige un volumen, por lo que la escena se visualiza desde un plano tridimensional. En cambio, el ejemplo (a) conceptualiza el campo carente de vegetación espesa, es decir, lo conceptualiza de manera bidimensional. En la LC se usa el término granulosidad para referirse al grado de especificidad de descripción de una escena. Así, el ejemplo (a) es una representación poco detallada, es decir de grano grueso (coarse-grained), mientras que el ejemplo (b) ofrece una visión más detallada o de grano fino (fine-grained). En efecto, este tipo de ajuste escalar es cuantitativo porque implica los aspectos espaciales bi- y tridimensionales, es decir, una progresiva focalización de la escena, de un grano grueso a uno más fino.

El segundo ajuste focal es el de tipo cualitativo y corresponde a la noción de esquematización que Langacker (1987) incluye dentro de la categoría de abstracción. La esquematización, según este autor, es la concepción de algo haciendo uso de una categoría más amplia. Croft y Cruse (2004, p. 53) llaman a la esquematización ajuste escalar cualitativo, que es una operación en la que, a diferencia del ajuste focal cuantitativo, en el que se pierde una escala o dimensión mesurables, en este se pierden propiedades irrelevantes. Es decir, la operación de esquematización permite conceptualizar algo a través de una categoría más amplia que la engloba. Por ejemplo, animal [A] es más esquemático que perro [B] y este último es una elaboración o instancia (instance) de animal [A], ya que todos los rasgos característicos de animal [A] están presentes en perro [B]. Dicho de otro modo, la entidad $[\mathrm{A}]$ es más esquemática respecto a [B] porque es menos detallada, 
mientras que $[\mathrm{B}]$ contiene los rasgos de $[\mathrm{A}]$ y especificaciones más granulares $\mathrm{y}$, por lo tanto, es una elaboración de [A].

Así las cosas, la esquematización forma parte del fenómeno de la atención porque el hablante puede hacer uso de conceptos más o menos esquemáticos atendiendo o haciendo caso omiso a ciertos aspectos de perfiles o de marcos.

\subsubsection{Juicio/comparación: categorización y figura/fondo}

La mente humana, para comprender y almacenar información, se sirve de la comparación de experiencias, entidades o conceptos conocidos con otros nuevos, así como de un juicio para poder designar las nuevas experiencias o conceptos a una determinada clase. Las operaciones de categorización, figura/fondo y metáfora forman parte de la habilidad cognitiva básica del juicio/comparación (Croft \& Cruse 2004), ya que, categorizar significa enmarcar una determinada situación mediante su comparación con otra(s). La metáfora consiste en una comparación y asociación de dos dominios cognitivos y la operación figura/fondo implica el contraste de la prominencia focal de dos participantes dentro de una misma escena. Aun cuando la metáfora forma parte de las operaciones de juicio/comparación, consideramos necesario tratarla como acápite aparte para poderla exponer con más detalle debido a la relevancia que tiene para esta investigación.

\section{A. La categorización}

Como ya hemos mencionado, la LC sostiene que la mente humana entiende el mundo no solo en términos de entes individuales sino, sobre todo, en términos de categorías cognitivas, que se han definido como conceptos almacenados que conforman el lexicón mental, mediante los cuales se organiza la realidad. Por lo anterior, uno de los temas centrales de la LC es la categorización, debido a que se trata de un proceso mental básico que hace posible la comprensión del mundo y la producción lingüística. A diferencia de la semántica estructural, que ha dado explicación al proceso de categorización sosteniendo que una categoría se define a partir de los rasgos que la componen, los cuales son necesarios y suficientes, la LC, en cambio, sostiene que no existen categorías cerradas ni límites precisos entre ellas.

La teoría de la categorización comprendida por la LC parte de trabajos empíricos antropológicos y psicológicos que fueron la base para el desarrollo de la teoría de prototipos propuesta por Eleanor Rosch (1978). A través de diferentes experimentos, la psicóloga junto con su equipo de colaboradores demostró que no todos los miembros de una categoría la representan al mismo grado, sino que hay elementos más prototípicos y elementos progresivamente más periféri- 
cos. Asimismo, determinaron que el prototipo es el elemento que más atributos comparte con el resto de los miembros de la categoría y que la pertenencia de un elemento a la categoría depende del grado de similitud que tenga con el prototipo. Con todo, para Rosch, los prototipos no son miembros específicos, sino mejores ejemplos representantes de la categoría: "By prototypes of categories we have generally meant the clearest cases of category membership defined operationally by people's judgements of goodness of membership in the category" (1978, p. 196).

Por lo tanto, Rosch habla de efectos de prototipicidad, es decir, de asimetrías entre los miembros de una categoría considerados como mejores o peores representantes de esta. En este sentido, la pertenencia a una categoría es una cuestión de grado.

A partir de sus experimentos, Rosch (1978) llegó a varias conclusiones: en primer lugar, demostró que mientras más prototípico fuera un elemento, más alta era su frecuencia y el orden de mención en el discurso. En segundo lugar, observó que el orden y rapidez de aprendizaje de un elemento por parte de los niños depende de la mayor o menor cercanía que este tenga con el prototipo (mientras más similitudes tenga un elemento con el prototipo, más rápido será asimilado). Finalmente, Rosch comprobó que la velocidad con la que un elemento se asigna a una categoría depende también de su centralidad dentro de la categoría (1978, p. 13).

La psicóloga concibe las categorías como entidades difusas donde la centralidad de un elemento dependerá del número de rasgos que posea con respecto al prototipo y donde los miembros periféricos son el puente que conecta con otras categorías. Asimismo, retoma en su teoría la noción de semejanza de familia (al. Familienähnlichkeit) propuesta por Wittgenstein en Philosophische Untersuchungen (1953). Esta noción se basa en la idea de que no todos los miembros de una categoría deben compartir todos los rasgos definitorios de esta, sino que pueden tener solo alguno o compartir algún rasgo con algún(os) miembro(s) (Rosch \& Mervis 1975).

La teoría de prototipos postulada por Rosch no pretende dar explicación sobre qué causa los efectos de prototipicidad. Sin embargo, Lakoff responde a esta interrogante a través de la teoría de los MCI:

[. . .] we organize our knowledge by means of structures called idealized cognitive models, or ICMs, and [. . .] category structures and prototype effects are by-products of that organization (1987, p. 68).

Así pues, Lakoff (1987) considera que el prototipo es la imagen mental definida a partir de un modelo cognitivo idealizado. Asimismo, afirma que los efectos de prototipicidad dependerán del grado de coincidencia entre los rasgos de un deter- 
minado elemento y los aspectos del MCI que caracterizan al prototipo (1987, p. 71). En este sentido, un ente se encontrará más al centro de la categoría mientras más rasgos comparta con el MCI que define al prototipo.

\section{B. La alineación figura/fondo o trajector/landmark}

La operación de conceptualización figura/fondo (ing. figure/ground) deriva de la psicología de la Gestalt y está muy relacionada con la percepción visual. Talmy $(1983,2000)$ introduce esta operación dentro de la LC para explicar la manifestación de las relaciones espaciales en el lenguaje natural. Sostiene que todas las relaciones espaciales se expresan a partir de la especificación de la posición de un objeto, la figura, en relación con otro, el fondo. La figura se distingue por ciertas características prototípicas: posee una localización menos conocida, es un elemento más pequeño que el fondo, más móvil, estructuralmente más simple, más prominente y más reciente en la conciencia. En contraste, el fondo es el elemento cuya localización es más conocida, es más grande que la figura, más estático, estructuralmente más complejo y se encuentra en el segundo plano de la escena (Talmy 1983, pp. 230-231).

En muchos casos la relación entre figura y fondo es simétrica y la elección entre (a) [El libro $]_{\mathrm{FIGUA}}$ está junto al [lápiz $]_{\mathrm{FoNDO}} \mathrm{y}$ (b) [El lápiz $]_{\mathrm{FIGURA}}$ está junto al [libro $]_{\text {FonDo }}$ depende únicamente del elemento que pongamos en el foco. Sin embargo, hay casos en los que la relación figura/fondo es asimétrica y los elementos no son intercambiables, sobre todo si la relación es entre elementos móviles y más pequeños versus elementos más grandes y estables como en (c) (Talmy 2000, p. 314):

(c) La bicicleta está cerca de la casa. vs. *La casa está cerca de la bicicleta.

La alineación figura/fondo se puede aplicar también a la relación entre sucesos. Es el caso de algunas frases subordinadas que fungen como fondo (causa o precondición) para la figura que se encuentra en la frase principal. También aquí, la relación es asimétrica - ej. (d) - en contraste con la relación simétrica que se establece en las frases coordinadas -ej. (e) - y muchas veces los elementos no pueden ser intercambiables - ej. (f) - (Talmy 2000, p. 325):

(d) Ana cocinaba mientras Juan veía la TV.

(e) Ana cocinaba y Juan veía la TV

(f) Soñó mientras dormía. vs. *Durmió mientras soñaba. 
Langacker (1987, p. 217; 2008, pp. 70-73) usa las nociones trajector (tr.) y landmark (lm.) para referirse a figura y fondo respectivamente. La alineación trajector/landmark es entendida en términos de prominencia focal primaria y secundaria. En este sentido, el trajector es el participante más prominente, al que se localiza, evalúa o describe (Langacker 2008, p. 70). En contraste, el landmark es el participante que posee un focus secundario (Langacker 2008, p. 70). Es llamado así porque prototípicamente provee puntos de referencia para la localización del trajector (Langacker 1987, p. 217).

Langacker explica que las expresiones pueden tener el mismo contenido y perfilar la misma relación, pero difieren en significado dependiendo de qué participante se elija como trajector y como landmark (2008, p. 70). Así, por ejemplo, en las oraciones $(\mathrm{g})$ y $(\mathrm{h})$, las locuciones preposicionales encima de y debajo de indican la localización espacial de dos objetos con respecto al eje vertical y perfilan la misma relación referencial (X encima de Y presenta la misma relación que $\mathrm{Y}$ debajo de $\mathrm{X}$ ), pero hay un contraste semántico que reside en el grado de prominencia conferido a los participantes (2008, p. 71):

(g) [La lámpara $]_{T R}$ está encima de [la mesa. $]_{L M}$

(h) [La mesa $]_{T R}$ está debajo de [la lámpara. $]_{L M}$

Prototípicamente, las funciones de sujeto y objeto reflejan la relación trajector/ landmark. Pero estas dos nociones pueden ser aplicables a cualquier tipo de dominio cognitivo (2008, p. 72), por ejemplo, las relaciones temporales entre distintos eventos también pueden ser conceptualizadas en términos de trajector $\mathrm{y}$ landmark. Así, por ejemplo, en las oraciones (i) y (j) tanto antes como después perfilan una relación de precedencia temporal entre dos eventos, pero el contraste semántico radica en la elección del evento trajector y del evento landmark (2008, p. 72):

(i) $[\text { Todos los invitados se habían ido }]_{T R}$ antes de que [llegáramos. $]_{L M}$

(j) [Llegamos $]_{T R}$ después de que [los invitados se habían ido. $]_{L M}$

Las nociones de figura/fondo propuestas por Talmy (1983; 2000) y las de trajector/landmark desarrolladas por Langacker (1987) se refieren a los mismos conceptos teóricos: ambos pares implican el contraste de la prominencia focal de dos participantes dentro de una misma escena. 


\subsubsection{La perspectiva/situacionalidad: subjetividad y objetividad}

La perspectiva es otro de los procesos cognitivos básicos del ser humano y tiene que ver con el punto de vista desde el cual es conceptualizada una escena. La localización y orientación espaciales en las que nos encontremos determinará la conceptualización de la situación que deseamos comunicar. Por esta razón, la perspectiva es fundamental para la construcción de imágenes (imagery) (Maldonado 2012, p. 230). Dentro de esta capacidad cognitiva, Langacker (1987, p. 132) recoge las operaciones de subjetividad/objetividad. Tales nociones hacen referencia a la posición que asume el conceptualizador, ya sea dentro o fuera de la escena. Un ejemplo muy claro de objetividad se encuentra en el llamado baby talk donde la madre dice al niño frases como estas: "mamá te trajo un regalo” o “¿cómo está mi niño?” En ambos casos los deícticos yo (mamá) y tú (niño) se objetivizan y el hablante/conceptualizador se ve a sí mismo como si fuera otro individuo, es decir, se coloca fuera de la escena. En cambio, si el hablante (la madre) conceptualiza la situación de manera subjetiva, es decir, se ubica dentro de la escena, usaría los pronombres personales deícticos para definir su identidad y la de su interlocutor (el niño) en relación con el acto de habla: “te traje un regalo”, “¿cómo estás?”.

\subsubsection{La metáfora}

En las teorías lingüísticas clásicas, la metáfora es considerada una cuestión de la lengua, un mecanismo propiamente literario, ajeno al lenguaje cotidiano convencional. En otras palabras, el término metáfora hace referencia a una expresión literaria o poética en la cual una o más palabras que pertenecen a un cierto concepto son usadas fuera de su uso convencional para expresar un concepto similar (Lakoff 2006, p. 185). En contraste, para la LC las generalizaciones que rigen las expresiones metafóricas no pertenecen al lenguaje, sino al pensamiento: la metáfora es un proceso cognitivo que permea el lenguaje (Lakoff 2007, p. 185).

Uno de los trabajos más influyentes sobre la metáfora dentro de la LC ha sido el llevado a cabo por George Lakoff y Mark Johnson. En su libro Metáforas de la vida cotidiana (1986) (originalmente en inglés Metaphors We Live By, 1980), a través de una gran cantidad de ejemplos demuestran que las metáforas forman parte de la forma ordinaria y cotidiana en que hablamos, pensamos y, por lo tanto, influyen en la manera en que actuamos.

De acuerdo a su función, los autores distinguen entre metáforas estructurales, ontológicas y orientacionales. En las primeras, un concepto, una actividad o 
experiencia se estructura en términos de otro/otra (Lakoff \& Johnson 1986, p. 50). Ejemplo de este tipo de metáforas es EL AMOR ES UN VIAJE, ${ }^{4}$ de la cual surgen expresiones metafóricas como Nuestro noviazgo va sobre ruedas; o EL TIEMPO ES UN RECURSO (VALIOSO), metáfora reflejada en la expresión metafórica El tiempo es dinero. La función de este tipo de metáforas consiste en organizar nuestro conocimiento de un concepto a través de la estructura claramente delineada de otro concepto (Lakoff \& Johnson 1986, p. 101).

Las metáforas ontológicas son aquellas que dan un estatus de ente físico a un dominio de la experiencia de carácter abstracto. Este tipo de metáforas nos permiten referir, categorizar, agrupar y cuantificar nuestras experiencias y, de este modo, podemos razonar sobre ellas (1986, p. 63). Dentro de las metáforas ontológicas se encuentran (a) las metáforas de sustancia y entidad como LA MENTE ES UNA MÁQUINA: Voy a perder el control o Le falta un tornillo (1986, p. 66); (b) las metáforas de recipiente como LOS CAMPOS VISUALES SON RECIPIENTES: Ahora está fuera de mi vista; y (c) la personificación que nos permite "dar sentido a fenómenos del mundo en términos humanos -términos que podemos entender sobre la base de nuestras propias motivaciones, objetivos, acciones y características-.” (1986, p. 72): La inflación ha dado a luz una generación de gente preocupada por el dinero (1986, p. 72).

Las metáforas orientacionales surgen de nuestra constitución física, de nuestra experiencia corpórea y cultural, y estructuran una gran cantidad de conceptos en términos espaciales (Lakoff y Johnson 1986, p. 50). La mayoría tiene que ver con las orientaciones arriba-abajo, dentro-fuera, delante-detrás, profundo-superficial, central-periférico. Ejemplos de metáforas orientacionales son FELIZ/BUENO ES ARRIBA - TRISTE/MALO ES ABAJO: Eso me levantó el ánimo. Caí en una depresión (1986, p. 51); CONTROL/PODER ES ARRIBA - AUSENCIA DE CONTROL/ PODER ES ABAJO: Tengo control sobre ella. Está bajo mi control (1986, p. 52); LO CONSCIENTE ES ARRIBA - LO INCONSCIENTE ES ABAJO: ¡Arriba! (¡despierta! ingl. Wake up!). Se hundió en un profundo sueño.

Lakoff y Johnson indican también una distinción entre las metáforas según su grado de convencionalidad. Diferencian entre metáforas convencionales por un lado y metáforas imaginativas y de nueva creación por el otro. Las convencionales son "metáforas que estructuran el sistema conceptual ordinario de nues-

\footnotetext{
4 Para Lakoff y Johnson, el fenómeno lingüístico metafórico involucra tanto el nivel conceptual como el verbal. En este sentido, distinguen la METÁFORA, que es una actividad cognitiva, de la expresión metafórica, que se refiere a la expresión lingüística de esta actividad (Lakoff \& Johnson 1998, p. 44). Así, lo que para la teoría clásica es una metáfora, para la LC será una expresión metafórica. Usaremos versalitas para las metáforas y las cursivas para las expresiones metafóricas.
} 
tra cultura, que se refleja en el lenguaje cotidiano” (1986, p. 181). Estas metáforas se originan en las experiencias humanas de carácter físico y cultural, es decir, están basadas en esquemas de imagen. Los esquemas de imagen son estructuras de conocimiento abstracto que representan la experiencia en dominios básicos (cfr. Langacker 1987) y surgen de nuestra experiencia sensorial y corpórea. Ejemplos de esquemas de imagen son espacio, contención, locomoción, balance, fuerza, unidad, multiplicidad, existencia (Evans 2007, pp. 107-108). Las metáforas convencionales, pues, al emerger de representaciones esquemáticas no presentan detalles imaginativos precisos (Lakoff \& Turner 1989, p. 91). Dentro de este tipo de metáforas se encuentran las orientacionales, ontológicas y muchas estructurales.

Las metáforas de nueva creación son aquellas que están fuera de nuestro sistema conceptual convencional (Lakoff \& Johnson 1986, p. 181). Al igual que las metáforas convencionales, proporcionan una estructura coherente a nuestra experiencia destacando ciertos aspectos, desfocalizando otros y ocultando otros más (1986, p. 194). Por ejemplo, la metáfora EL AMOR ES UNA OBRA DE ARTE EN COLABORACIÓN focaliza la noción de TRABAJO pero no toma en cuenta la noción de VIAJE o de LOCURA que forma parte del marco experiencial del amor (1986, p. 194). Dentro de las metáforas de nueva creación se encuentran las metáforas de imagen (Lakoff \& Turner 1989, pp. 89-96) las cuales implican la correlación biunívoca entre imágenes mentales - convencionales o no- que coinciden en forma y en ciertos atributos. En otras palabras, las metáforas de imagen "are "one shot” metaphors: they map only one image onto one other image” (Lakoff 2006, p. 215). ${ }^{5}$ Así, por ejemplo, la expresión metafórica Mi mujer . . . cuya cintura es un reloj de arena sugiere una imposición de la imagen del reloj sobre la imagen de la cintura de la mujer en virtud de la similitud de su forma (Lakoff 2006, p. 215; Lakoff \& Turner 1989, p. 90).

\section{A. La teoría de la metáfora conceptual}

Para Lakoff (2006, p. 185), la metáfora es un proceso mental en el que se conceptualiza un dominio mental en términos de otro. Este proceso se realiza mediante el establecimiento de asociaciones o correspondencias (mapping) generales a través de dos dominios conceptuales. Tomemos como ejemplo la metáfora estructural EL AMOR ES UN VIAJE, que es la base para la conceptualización de expresiones metafóricas como No creo que esta relación vaya a ninguna parte o Tendremos

5 Trabajo originalmente publicado en 1993: Lakoff, George (1993). The contemporary theory of metaphor. En A. Ortony (ed.), Metaphor and Thought (pp. 202-251). Cambridge: Cambridge University Press. 
que emprender caminos separados. Tal metáfora implica la comprensión de un dominio de la experiencia, es decir, EL AMOR, en términos de otro dominio, EL VIAJE. De acuerdo con el autor, a nivel conceptual se establece una correspondencia ontológica (una asociación, mapeo o proyección, en ing. mapping) entre entidades que pertenecen a un dominio mental de origen o fuente (source domain) que presta sus conceptos (en el caso del ejemplo, EL VIAJE) y otra entidad que pertenece a un dominio destino o meta (target domain), generalmente más abstracto (en nuestro caso EL AMOR).

Para facilitar la comprensión del proceso metafórico, Lakoff y Johnson (1980; Lakoff 2006, p. 190) proponen una estrategia para designar la correspondencia: EL DOMINIO META es EL DOMINIO FUENTE (el amor es un viaje) o alternativamente EL DOMINIO META como/en los términos del DOMINIO FUENTE (el amor en los términos de un viaje).

La metáfora conceptual es pues un mapeo o un conjunto de correspondencias a través de las cuales las entidades del dominio del amor corresponden sistemáticamente a las entidades del dominio del viaje: los amantes corresponden a los viajeros; la relación amorosa al vehículo; sus objetivos a los lugares de destino; las complicaciones a las dificultades del viaje.

Existen dos tipos de proyecciones por medio de las cuales se realiza el proceso metafórico: las correspondencias ontológicas y las correspondencias epistémicas (Lakoff 2006, p. 191). Las primeras vinculan elementos concretos entre los dominios de origen y destino (como en la metáfora EL AMOR ES UN VIAJE). Las correspondencias epistémicas en cambio, vinculan porciones de conocimiento enciclopédico- que nos permiten hacer inferencias (Soriano 2016, p. 98) que se proyectan del dominio fuente al dominio meta.

Las correspondencias que se establecen entre los dos dominios de la metáfora conceptual son siempre parciales, es decir, nunca se importa toda la información que tenemos del dominio fuente sobre el meta, sino que se proyecta la información coherente con la estructura del dominio meta, la cual consiste en un esquema de imagen. En otras palabras, el razonamiento que se realiza en el dominio meta está regulado por la estructura de imagen del dominio fuente (Croft \& Cruse 2004, p. 201). En esto consiste lo que Lakoff llama hipótesis de la invariabilidad (invariance hypotesis, Lakoff 2006, p. 199). ${ }^{6}$ Debido a la característica de parcialidad, un dominio puede servir de fuente para varios dominios meta y un dominio meta puede estar estructurado por varias fuentes. Además

6 Con respecto a la parcialidad de las proyecciones metafóricas Ibarretxe-Antuñano (1999) propone la Teoría de la selección de propiedades la cual sugiere que son solo algunas de las propiedades prototípicas del dominio fuente las que se proyectan al dominio meta. 
de la parcialidad, otra de las características de la metáfora conceptual es su unidireccionalidad, es decir que la estructura del dominio fuente se proyecta al dominio meta, pero no al contrario (Soriano 2016, p. 105). Es este último aspecto uno de los criticados y superados por otras propuestas teóricas como la teoría de la integración conceptual (Conceptual Blending Theory), que expondremos a continuación.

\section{B. La teoría de la integración conceptual (Conceptual Blending Theory)}

A partir de la teoría de la metáfora conceptual (desde aquí TMC) y la teoría de los espacios mentales (Fauconnier 1994) (desde aquí TEM), Fauconnier y Turner (2002) desarrollaron la teoría de la integración conceptual (desde aquí TIC), que explica la conceptualización de distintos fenómenos lingüísticos entre los que se encuentra la metáfora. Esta teoría surge como alternativa para tratar fenómenos que ni la TEM ni la TMC podían explicar adecuadamente (Evans \& Green 2006, p. 400). La TIC explica la construcción de significado a partir de la integración de estructuras mentales - la cual no debe ser entendida como la suma de las partes de tales estructuras, sino como la creación de una red integrada que genera nuevos significados y estructuras inferenciales-.

El proceso conceptual de la integración o fusión tal como lo proponen Fauconnier y Turner (2002) consta de, mínimo, dos espacios mentales de entrada (input space 1 e input space 2), un espacio genérico (ing. generic space) y un espacio amalgamado o fusionado (blended space), como se observa en la Fig. 1.

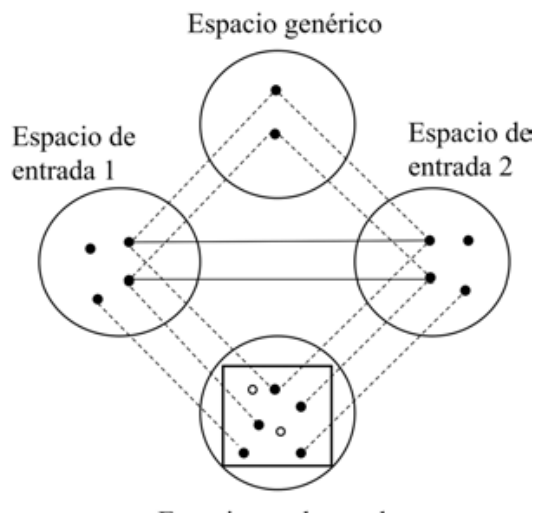

Espacio amalgamado
Fig. 1: Diagrama básico de la integración conceptual (basado en Fauconnier \& Turner 2006, p. 313).

Según expone Fauconnier (1994; 1997), los espacios mentales son dominios cognitivos parciales mediante los cuales vamos estructurando y dando sentido a lo 
que enunciamos y escuchamos. Por lo tanto, son estructuras que se generan online, esto es, sobre la marcha del discurso:

Mental spaces are small conceptual packets constructed as we think and talk, for purposes of local understanding and action. Mental spaces are very partial assemblies containing elements, and structured by frames and cognitive models. They are interconected, and can be modified as thought and discourse unfold. Mental spaces can be used generally to model dynamical mappings in thought and language (Fauconnier \& Turner 2006, p. 307). ${ }^{7}$

En efecto, dichas estructuras sirven para conceptualizar situaciones, objetos, entidades, acciones, procesos y periodos de tiempo; son escenarios que contienen información específica derivada de nuestro saber lingüístico, pragmático y cultural. Así descritos, los espacios mentales coinciden con los modelos de situación de van Dijk (1993, p. 41; 2011, p. 21; 2012, p. 108, cfr. supra) y, como estos, operan en la memoria a corto plazo o memoria de trabajo (Fauconnier \& Turner 2002, p. 103). Sin embargo, como lo indica la cita precedente, son construidos a partir de la activación de marcos y modelos cognitivos almacenados en la memoria a largo plazo y pueden formar parte de ella mediante procesos de fijación (Fauconnier \& Turner 2002, pp. 102-103; van Dijk 1985, p. 63).

Como sugiere el diagrama básico (Fig. 1), el proceso de integración conceptual consta de cuatro espacios mentales. Los dos espacios de entrada comparten conexiones (representadas en el diagrama por las líneas continuas) entre elementos homólogos. Las conexiones o relaciones vitales entre los elementos de los espacios de entrada pueden ser de distinta naturaleza: relaciones de identidad, de representación, de rol, de intencionalidad, causa-efecto, analogía (como en el caso de la metáfora), tiempo, espacio y categoría (Fauconnier \& Turner 2006, p. 313). Las líneas punteadas que conectan los elementos de los cuatro espacios mentales representan las proyecciones conceptuales a través de la red.

Además de dos (o más) espacios mentales de entrada, el proceso de integración conceptual implica un espacio genérico que contiene la estructura y los elementos que los espacios de entrada tienen en común. Estos elementos pueden ser reconocidos en términos de agentes, tajektor y landmark (Langacker 1987, 2008), acción, proceso. tiempo, etc. La noción de espacio genérico, sin embargo, ha sido cuestionada (cfr. Maldonado 1999). Los elementos que se identifican dentro de este espacio representan los aspectos o propiedades que los elementos de los dos espacios de entrada comparten. No obstante, consideramos innecesaria su mención, ya que son muy esquemáticos en el sentido

7 Este texto aparece originalmente en Fauconnier \& Turner (1998): “Conceptual integrations networks", Cognitive Science 22(2), 133-187. 
langackeriano (Langacker 2008, p. 19), es decir, su grado de especificación y detalle es muy bajo (p. ej., agente, tema, acción, tiempo, etc.). Además, tales elementos resultan redundantes, pues el tipo de conexiones que indican se puede dilucidar perfectamente sin recurrir a la especificación en un tercer espacio. Por esta razón, para el análisis que aquí nos ocupa, prescindiremos de este componente.

El cuarto espacio es el espacio de la integración (blended space). Este hereda la estructura parcial de los dos espacios de entrada y genera una estructura inferencial nueva o emergente. De acuerdo con Fauconnier y Turner (2006, pp. 314-315), la estructura emergente surge a partir de tres procesos: composición, complementación y elaboración. La composición es la proyección selectiva de la información de los espacios de entrada al espacio de integración. La composición de estos elementos proporciona relaciones que no existían en los espacios de entrada independientes. La complementación es la operación mediante la cual se recuperan conocimientos pertenecientes a la memoria a largo plazo como marcos cognitivos - o información proveniente del contexto para completar la información recibida. Por último, la elaboración es el trabajo cognitivo a partir del cual surge la estructura emergente. Dicho trabajo cognitivo dentro del espacio amalgamado implica el razonamiento, la producción de inferencias, el desarrollo de emociones, etc.

Como se puede observar, la TMC y la TIC son dos operaciones cognitivas que actúan de manera distinta. La TMC es una teoría sobre la organización conceptual (Hart 2010, p. 113), es decir, que la metáfora conceptual forma parte de las estructuras que se encuentran en la memoria a largo plazo de manera offline. Por el contrario, la TIC es una teoría de la conceptualización durante el discurso (2010, p. 114; Koller 2004, p. 8), o sea, se trata de procesos online que incorporan información de distinta índole que dan como resultado estructuras inferenciales nuevas. Debido al mapeo unidireccional que supone la TMC, no se considera que la estructura a nivel de marcos asociada con el dominio meta pueda contribuir al significado de la metáfora. En la TIC ambos espacios de entrada contribuyen al significado emergente. Grady et. al (1999, p. 101) refieren además que la TMC establece relaciones únicamente entre dos dominios, mientras que la TIC lo hace con más de dos. Esto es, explican que la TMC trata la metáfora como una representación conceptual en la que se establecen relaciones entre un dominio fuente y uno meta, mientras que la TIC trata la metáfora como una operación de conceptualización dinámica que implica, al menos, cuatro espacios mentales (1999, p. 101). Sin embargo, a pesar de actuar de manera distinta, estas teorías pueden ser compatibles y complementarias (Grady et al. 1999, p. 120), ya que las metáforas conceptuales se generan en espacios mentales, posterior- 
mente se fijan en la memoria a largo plazo y, como los marcos, se evocan en procesos de conceptualización como la integración conceptual.

\subsubsection{Dinámica de fuerzas}

Otra de las operaciones de conceptualización que Croft y Cruse (2004) categorizan dentro de la capacidad cognitiva básica de constitución o Gestalt (forma) es la dinámica de fuerzas (force dinamics). El modelo de la dinámica de fuerzas propuesto por Talmy $(1988 ; 2000)$ da cuenta de las relaciones de causa-efecto en términos de fuerza, presión y movimiento (2000, p. 409). Es decir, este modelo toma en cuenta las relaciones de fuerza que se establecen entre las entidades físicas: ya sea de fuerza, de presión, de resistencia, de bloqueo y de eliminación del bloqueo.

Este sistema dinámico comprende dos entidades que ejercen fuerza: el agonista, que es la entidad en la que se enfoca la atención, y el antagonista, la entidad que se opone al agonista, ya sea superando su fuerza o fracasando en superarlo. La fuerza intrínseca del agonista puede ser hacia el movimiento o hacia el reposo (acción o inacción), y la fuerza intrínseca del antagonista resulta opuesta a esta tendencia. La entidad que es capaz de manifestar su tendencia a costa de su oponente es la entidad más fuerte (Talmy 2000, p. 414). Así pues, dependiendo del tipo de relación de fuerza que se establece, el agonista es libre de llevar a cabo su tendencia intrínseca o es bloqueado por el antagonista. Talmy (1988, p. 54; 2000, p. 414) utiliza los siguientes diagramas para representar los elementos de la dinámica de fuerzas (Fig. 2):
a. Elementos
b. Tendencia intrínseca
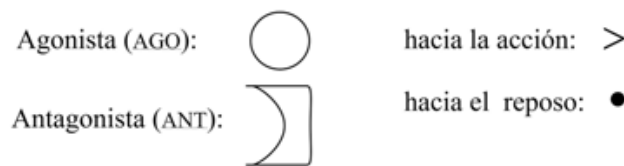

c. Resultado de la interacción de fuerzas

d. Balance de fuerzas

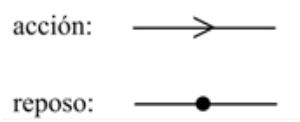

Entidad más fuerte: +

Entidad más débil: -

Fig. 2: Elementos de la dinámica de fuerzas (basado en Talmy 2000, p. 414). 
La dinámica de fuerzas se expresa mediante preposiciones y conjunciones (close class gramatical items) así como por elementos léxicos que no solo refieren a las interacciones físicas de fuerza, sino, por extensión metafórica, a las relaciones e interacciones psicológicas y sociales conceptualizadas en términos de presión (Talmy 2000, p. 409): verbos - principalmente modales con valor deóntico y epistémico-, sustantivos, adjetivos, adverbios, interjecciones (open class lexical items).

Talmy plantea cuatro posibles patrones de estado estacionario (steady-state patterns 2000, p. 415), dos de los cuales son de tipo causal (a. y d.) (Fig. 3).

a.

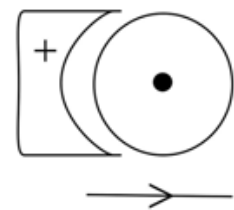

c.

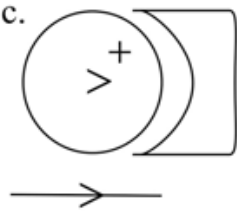

b.

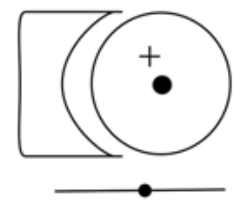

d.

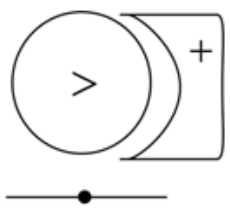

Tendencia del AGO:

$(a, b)$ : hacia el reposo

(c, d): hacia la acción

Resultado para el AGO: $(\mathrm{a}, \mathrm{c})$ : acción

(b, d): reposo

Fuerza del AGO frente a la del ANT:

(a, d): menor

$(b, c)$ : mayor

Fig. 3: Patrones básicos de estados estacionarios en la dinámica de fuerzas (basado en Talmy 2000, p. 415).

Asimismo, explica la dinámica de fuerzas mediante los siguientes ejemplos (2000, p. 416) (donde AGO está por agonista y ANT por antagonista): ${ }^{8}$

(a) [La pelota $]_{\mathrm{AGO}}$ siguió rodando debido [al viento $]_{\mathrm{ANT}}$ que soplaba sobre ella.

(b) $[\mathrm{El} \text { cobertizo }]_{\mathrm{AGO}}$ permaneció en pie a pesar del fuerte [viento $]_{\mathrm{ANT}}$ que soplaba contra él.

8 La traducción y la indicación de agonista/antagonista es nuestra. En el original:

a. The ball kept rolling because of the wind blowing on it.

b. The shed kept standing despite the gale wind blowing against it.

c. The ball kept rolling despite the stiff grass.

$\mathrm{d}$. The log kept lying on the incline because of the ridge there. 
(c) [La pelota $]_{\mathrm{AGO}}$ siguió rodando a pesar de [la hierba $]_{\mathrm{ANT}}$ áspera.

(d) $[\mathrm{El} \text { tronco }]_{\mathrm{AGO}}$ se mantuvo inmóvil en la pendiente debido a [la cresta de la montaña $]_{\mathrm{ANT}}$.

En (a) la tendencia del agonista [la pelota] es hacia el reposo, pero es superado por el antagonista [el viento] que tiende al movimiento y es más fuerte, por lo que supera la resistencia del agonista y lo obliga a moverse. Por lo tanto, el antagonista se encuentra en una relación causal con el agonista. Esta oración se puede ilustrar mediante el patrón de estado estacionario a. (Fig. 3). El patrón de estado estacionario b. (Fig. 3) corresponde al ejemplo (b) donde la tendencia de la fuerza del agonista [el cobertizo] es también hacia el reposo, pero, a diferencia del primer ejemplo, su fuerza es mayor a la del antagonista, por lo que logra manifestar su tendencia estática. En el tercer ejemplo, que corresponde al patrón c. (Fig. 3), la tendencia de la fuerza del agonista [la pelota] es hacia el movimiento, y aunque la fuerza del antagonista [la hierba] se opone a él, su fuerza es mayor, logrando así manifestar su tendencia intrínseca. En el último ejemplo (d), representado por el cuarto patrón de la dinámica de fuerzas (d., Fig. 3), la tendencia de la fuerza del agonista, [el tronco], también es hacia el movimiento, pero esta vez la fuerza contraria del antagonista, [la cresta de la montaña], es mayor e impide el movimiento. Este patrón, como el de a., es de tipo causal. En los ejemplos anteriores, la interacción de la dinámica de fuerzas se expresa mediante las locuciones preposicionales debido a y a pesar de, así como por los verbos seguir o permanecer/mantenerse. Por un lado, debido a indica la mayor fuerza del antagonista, que supera la fuerza del agonista y por lo tanto implica causalidad. En cambio, a pesar de codifica la mayor fuerza del agonista frente a la debilidad del antagonista. Por otro lado, los verbos seguir, continuar, mantenerse (en ing. keeping) son indicadores de la presencia de dinámica de fuerzas (Talmy 2000, p. 417).

Además de los cuatro patrones de estado estacionario, Talmy identifica distintos patrones de cambio de dinámica de fuerzas. Entre estos se encuentran los patrones de cambio del estado de fuerza (shift in state of impingement) (2000, p. 417) y de cambio de balance de fuerza (shift in balance of strength) (2000, p. 419). En los cuatro patrones de cambio del estado de fuerza que Talmy identifica (Fig. 4), el antagonista no aplica al agonista una presión continua, sino que entra o abandona el estado de presión.

En este tipo de patrones, el antagonista es el elemento más fuerte. La flecha que apunta hacia abajo indica que abruptamente se aplica una fuerza, mientras que la flecha apuntando hacia arriba significa que el antagonista suelta la fuerza ejercida, con lo que permite al agonista manifestar su tendencia intrínseca. 
e.

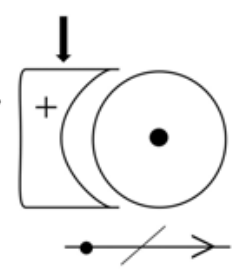

g.

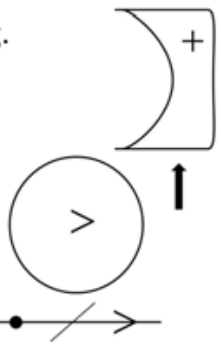

f.

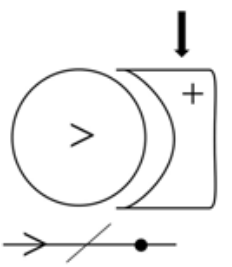

Tendencia del AGO:

(e, h): hacia el reposo

(f, g): hacia la acción

Efecto del ANT: $\quad(e, f)$ : causar

$(\mathrm{g}, \mathrm{h})$ : liberar

Resultado para el AGO: $(\mathrm{e}, \mathrm{g})$ : iniciar

$(\mathrm{f}, \mathrm{h})$ : detener

Fig. 4: Patrones de cambio de estado de fuerza (basado en Talmy 2000, p. 418).

Además de ser el más fuerte, el antagonista es considerado en primer lugar y normalmente toma la posición de sujeto, como se observa en los siguientes ejemplos: ${ }^{9}$

(c) $[\text { El golpe }]_{\mathrm{ANT}}$ de la pelota hizo que la [lámpara $]_{\mathrm{AGO}}$ cayera de la mesa.

(d) $[\mathrm{El} \text { agua }]_{\mathrm{ANT}}$ que gotea hizo que [el fuego $]_{\mathrm{AGO}}$ se apagara.

(e) $[\text { El tapón }]_{\mathrm{ANT}}$ se aflojó y ahora deja pasar [el agua $]_{\mathrm{AGO}}$.

(f) $[\text { La ruptura }]_{\mathrm{ANT}}$ del agitador hace que [las partículas $]_{\mathrm{AGO}}$ se asienten.

En el primer ejemplo, correspondiente al patrón e., el agonista tiene tendencia al reposo, pero la fuerza aplicada por el antagonista hace que cambie su estado y se provoque el movimiento. En (f), el antagonista, que es el participante más fuerte, entra en choque contra el agonista - el cual tiende al movimiento y se ha estado moviendo- y lo detiene. En (g), el antagonista (participante más fuerte) ha estado

9 e. The ball's hitting it made the lamp topple from the table.

f. The water's dripping on it made the fire die down.

g. The plug's coming loose let the water flow from the tank.

h. The stirring rod's breaking let the particles settle. (Talmy 2000, p. 418) 
bloqueando al agonista (que tiende al movimiento), pero lo suelta y este último puede manifestar su tendencia intrínseca. Por último, en (h) un antagonista que se mantiene enérgicamente en movimiento detiene su fuerza $\mathrm{y}$, con esto, permite al agonista, que tiende hacia el reposo, volver a su tendencia original.

En suma, Talmy (2000, p. 418) reconoce dos tipos de estados causales: los patrones donde el antagonista aplica una presión continua (extended causation), como en los ejemplos (a) y (d); y los patrones en los que la presión se aplica una vez y causa un cambio de estado, ya sea de movimiento o reposo (onset causation) (ejemplos [e] y [f]).

Por lo que se refiere al cambio de balance de fuerza, Talmy (2000, p. 419) explica que el balance de la fuerza entre el antagonista y el agonista cambia por el fortalecimiento o debilitamiento de una de las entidades:

i) $[\text { El enemigo }]_{\mathrm{ANT}}[\mathrm{nos}]_{\mathrm{AGO}}$ venció mientras estábamos defendiendo la frontera.

Mientras que en los ejemplos (e), (f), (g) y (h) el antagonista aplica o abandona la presión -con lo cual inicia o termina su efecto dominador-, en el ejemplo (i) el antagonista no llega sino que se vuelve más fuerte o más débil. Mediante la flecha curva que se observa en la figura 5 se representa este cambio en la fuerza: ${ }^{10}$

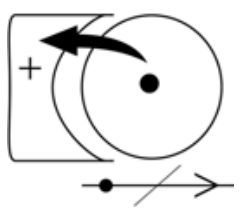

Fig. 5: Cambio de balance de fuerza (basado en Talmy 2000, p. 420).

10 Esta flecha indica el cambio de fuerza, pero no representa la transferencia de la fuerza de una entidad a otra. 
